possibility of spontaneous cure lies in the successful expulsion of the stones through the biliary passages, or in the successful ulceration into the alimentary canal,- - or, in very rare instances, in the successful ulceration through the abdominal wall.

The foregoing statement of facts concerning the spontaneous cure of offending gallstones includes the only argument of importance against surgical intervention. But we have, under the most favorable circumstances, painful, perhaps often repeated and prolonged, colics, with a strong probability that they will continue indefinitely, with no certainty of permanent cure, - with every probability that with increasing age of the patient and increasing size of the stone, the difficulties and dangers of passage will also increase.

We have on one hand the painful, dangerous and uncertain processes of ulceration,-- processes which may require years for their completion, and which invite the disasters of localized infection, biliary extravasation, general peritonitis, faulty adhesions, liver and pancreatic infections, and new growths, - the clumsy, dangerous and ineffectual methods of nature. We have on the other hand the safe, rapid, intelligent and scientific work of a few minutes, with absolute demonstration, not only that the gallstones themselves have been removed, but that complicating conditions have, if possible, been relieved.

(6) There is also the possibility that, after offending enough to prove the diagnosis, gallstones may give no further trouble.

The possibility that gallstones which have once offended may never again offend cannot be denied. The fact that in a given case it is impossible to tell what the future will be makes this argument of little weight. If in any case there are distinct contra-indications to operation, the possibility that there will be no further trouble may be looked upon with a certain satisfaction; but, as an argument against intervention, this possibility should receive but little consideration.

(7) The last and decisive attack of biliary colic may have been caused by the last remaining gallstone, exploration showing that none remain.

This argument has already been discussed.

As time goes by, the difficulties and doubts which many of us felt in the beginning as to the best treatment of gallstones become dissipated in the light of renewed experience. Aided by numerous explorations upon the living, especially in cases of doubt, we are becoming more and more accurate in diagnosticating the lesion. We have demonstrated, by thousands of operations upon the abdominal cavity, the safety of simple explorations. At the same time we have demonstrated in hundreds of cases the pathological appearances of gallstone lesions in their earliest stages. We have seen the changes, after years of natural efforts at expulsion, in the thickened, contracted and friable gall bladder; in the gall bladder buried in extensive adhesions of the right upper quadrant, contracted upon large stones, ulcerated, and filled with septic fluids. We have seen the perforations from the gall bladder into the intestine, the peritoneal cavity, the abdominal wall; obstructions of the cystic, hepatic and common ducts; strictures at the duodenal papilla; biliary fistula; the acute infections of the gall bladder varying between simple distentions with mildly septic fluids and total gangrene of the gall bladder wall. We have seen the gall bladder distended with the chronic puriform fluids of long continued inflammation, or infected by the highly septic fluids of an acute cholecystitis. We have observed, only too often, malignant new growths of the interior of the gall bladder itself; cancer of the liver secondary to cancer of the gall bladder; acute and chronic infections of the pancreas. In a word, we have seen a great variety of lesions, owing directly or indirectly to the presence of gallstones, lesions which in the early stages would have been safely prevented by surgical treatment.

The spontaneous cure, whether or not it is aided by medicinal treatment, is clumsy, dangerous, ineffectual and contrary to common sense. Once gallstones begin to offend, there is no way of telling how the process will end, except that, on the chances, it will end either in permanent disability or in death. Treated mechanically, as they are by surgical art, gallstones are quickly, safely and permanently removed, at the least possible expense in suffering and in danger:

\section{OBSTRUCTIVE DISEASES OF THE LOWER BOWEL.1}

BY HENRY O. MARCY, M.D., BOSTON.

Tue diseases of the pelvic viscera, which culminate in obstructive interference of the function of the lower bowel and bring into consideration surgical measures for relief or cure, constitute one of the most interesting chapters in the history of pelvic diseases.

The pathologic changes incident to such conditions are, for the most part, chronic, and are usually accompanied by discomfort, pain and suffering of an extreme character. The limitation of time at my disposal absolutely prevents an exhaustive treatment of the subject. For the sake of convenience, it may be well to subdivide it : (1) The conditions extraneous to the bowel; (2) the obstruction caused by its contents; and (3) the pathologic conditions belonging to the viscus itself.

The changes incident to injuries of the pelvis, causing obstructive interference with the function of the lower bowel, are usually of an acute character and, as a consequent, do not come within the scope of the present discussion.

As the result of injuries, however, changes may supervene which materially impair the function of the lower intestine and, although not produc-

${ }^{1}$ Read by invitation before the Massachusetts Medical Society, June 11, 1901, as a part of the general topic, "In What Cases of Disease of the Alimentary Tract, Not Emergencies, Should OperaDisease of the Alimentary Tract, Not
tion be Advised for Relief or Cure?" 
ing a positive obstruction, demand operative intervention.

Fracture of the coccyx is a well-recognized illustration of such a condition. Deformation of the pelvis and osteoplastic growths also deserve mention.

In the male, diseases of the seminal vesicles and prostate frequently bring about such an irritated condition of the lower bowel as to induce most painful suffering and impair the function of defecation to a degree closely simulating intestinal obstruction. This is also true in old cases of stone in the bladder. Fortunately, owing to earlier surgical intervention, these conditions are far less common than formerly. Rarely chronic distention of the bladder may induce the most pronounced type of rectal irritation and be interpreted as a constant desire for defecation.

Incident to diseases of the reproductive organs in the female, interference of the function of the lower bowel to a degree demanding surgical intervention is by no means rare. The infectious diseases of the Fallopian tubes, with the secondary conditions dependent thereon, have, as a very common symptom, the disturbance of the intestinal function so as to produce the most acute suffering and, on this account, demand surgical intervention.

Small cystic tumors of the ovary or broad ligament not seldom bring about a train of functional disturbances of the lower bowel, seriously interfering with defecation, and occasionally 'by mechanical pressure make urgent demand for surgical relief. The retroversion of the gravid uterus sometimes produces the most marked tenesmus and straining, causing the patient, among other symptoms, an intense and unrelieved desire for defecation. Uterine tumors not seldom so block the pelvis as to arrest the function of the lower bowel, and for this reason render surgical intervention necessary. Unfortunately, this latter condition may come on so gradually as not to be suspected by the patient, and may be easily overlooked by the physician. Instances of this type are by no means rare. I have operated upon a number of patients where the impairment of the intestinal function was the more prominent symptom because of a small uterine myoma having become firmly wedged in the lower pelvic strait. An illustrative case of this type is the following: Miss H., age 58. Menstruation ceased about 10 years ago. Menorrhagia for some years was very pronounced. At 40 she suffered severely from weight and distention caused by a large symmetrical fibroid tumor, distending the abdomen almost to that of full term of pregnancy. This slowly lessened in size after the menopause until it was believed by herself and her physician to have entirely disappeared. Six months previous to operation, vesical tenesmus and intestinal obstruction became so marked that she was obliged to take her bed and was too sick to leave it, her life being despaired of. I removed, with the greatest of difficulty, a calcified uterine tumor almost completely filling the pelvis and so firmly wedged in the lower strait that it required nearly my entire strength, aided by the Trendelenburg position, to dislodge it. Months followed before the intestinal function was completely restored.

The pathologic changes which occur in the hemorrhoidal plexus of vessels are sometimes so pronounced as to require surgical treatment, not alone because of the local conditions of pain and suffering, but that, coassociated with dilatation of the rectum, obstructive conditions of the lower bowel supervene. I have several times met with displacement of the pelvic viscera, causing an arrest of intestinal function to such a degree as to demand operation. 'Two cases of this character were incident upon hernia of the bladder, one in which for some days intestinal obstruction had been complete. The patient's condition was in extremis incident upon an old hernia in which 8 or 10 inches of the large intestine had long been incarcerated in the sac.

The medical practitioner is not seldom called upon to relieve acute distress from the accumulation of foreign material lodged in the lower canal. This is an experience common to all practitioners, and yet it is occasionally of a type and character demanding surgical intervention. Fecal accumulations are by far the most common, and the suffering incident thereto is often intense. This is more frequent in women, owing to the pathologic conditions of her pelvic viscera, dependent upon the result of the loss of perineal support, following childbirth, impairing the function of the lower bowel and inducing rectocele.

This condition is, however, not seldom met with in men and in women without other pelvic complications.

Some time since, I operated upon a woman with a history of more than 30 days having passed without defecation. The suffering was less than might have been supposed, and yet the entire lower bowel was blocked with such a mass of dried feces that, after complete etherization, the accumulated material was removed with much difficulty. 'Two cases have been reported to me in which the period of nondefecation was yet longer, and a considerable number of such cases are on record. It is very probable, however, that these conditions have been preceded by a long period of comparative functional inaction of the lower bowel, with previous distention, etc.

Foreign bodies are by no means rarely found interfering with defecation, demanding the intervention of the surgeon. It is remarkable that an individual may swallow accidentally such articles without knowledge thereof until arrested by the sphincter muscle. Pieces of bone are probably the more common. On one occasion the most acute distress, demanding immediate relief, was caused by a sharp thorn more than an inch in length, which I removed after it had penetrated the lower border of the sphincter muscle. Spasmodic contraction of the muscle was almost continuous, producing the most intense suffering. The patient had no knowledge of its presence in the alimentary canal until thus caught in the act 
of defecation. Some time since a very intelligent lawyer brought me a round steel nail $1_{\frac{1}{2}}$ inches in length, bent at an angle of $45^{\circ}$. He was hastily eating in a railroad restaurant, almost at the same time, $a$ hot biscuit and a baked apple. He felt a slight scraping in the throat, which he attributed to swallowing the stem of the apple. He had no knowledge of the nail until caught by the sphincter muscle in the act of defecation, producing intense suffering. Some years ago two immense gallstones were brought me for examination, having been lodged in the lower bowel, from whence they were removed by the family physician. For months it had been believed that the patient was dying from cancer of the liver, so intense were her jaundice and the local suffering. Immediate relief was experienced, and the patient began slowly to improve when, nearly 6 weeks later, the removal of these gallstones, measuring over an inch in diameter, aided in making a more correct diagnosis. At the pathologic exhibit of the American Medical Association at St. Paul, in June, 1901, there was a calcareous concretion, socalled bowel stone, quite 3 inches in diameter, which had caused obstruction.

A most heterogenous collection of foreign materials are reported as having been removed from the rectum, many having been placed there by the sufferers themselves while not in stable mental condition.

The most important subdivision of the subject remains for consideration; namely, structural changes in the wall of the intestine, due to malignant growths. Since cancer of the intestine has been the subject of the two preceding essayists, it would be a fruitless repetition for me to enter into the discussion of the pathologic conditions, except so far as relates to its surgical aspect. In a recent paper by E. N. Mason, ${ }^{2}$ entitled "Some Remarks from an Analysis of 5,000 Cases of Malignant Disease," the author draws some very interesting deductions. Sixty-three per cent. of the cases were females. This is shown to be due to the greater tendency of cancer to occur in the breast and in the uterus. The demonstration is made that by excluding all the cases of malignant disease occurring in organs peculiar to either sex, cancer is more common in the male, in the proportion of about 53 to $47 \%$. This the author traces more especially to trauma and syphilis. About three-fourths of all the cases of cancer in the male occur in the alimentary tract. His tables show that, in $7 \%$ of the cases, the disease was located in the rectum, and that there were nearly twice as many males as females.

It is interesting to note that the committee, in discussing the predisposing causes of malignant disease, give the first place " to prolonged local irritation due to various causes, setting up inflammatory changes in the irritated tissues."

By far the greater emphasis is to be placed upon the early recognition of the malignant changes in the lower bowel, since in the treatment of cancer of the rectum little can be hoped

2 British Medical Journal, May 18, 1901. for except from prompt surgical intervention before the disease has deeply invaded the tissues. I cannot emphasize too strongly the importance of a careful examination of the lower bowel in a large class of cases which are generally overlooked by the family physician. Unfortunately, the great majority of the sufferers from cancer of the rectum come to the surgeon when it is too late to hope for cure by complete removal of the diseased structures. When cancer has advanced to come within the strict limitation of my subject - obstructive disease of the lower bowel - little can be hoped for except palliation by colostomy. The usual place of selection is below the left anterior superior iliac spine above Poupart's. ligament. In this operation, it is usually the better way to make a funnel-shaped incision through the abdominal wall, parallel to Poupart's ligament, commencing just below the spine of the ileum. The sigmoid flexure of the intestine is easily found and is carefully sutured to the peritoneum, the needle being made to penetrate the connective tissue coat of the intestine. Good surgeons vary in the method of suturing. I much prefer fixation by two lines of fine continuous tendon sutures. Unless imperative, the intestine should not be opened until after 24 hours, when peritoneal union will be found to have taken place. The division of the bowel is best effected with the cautery knife. The mucous membrane may be drawn out and sutured to the skin, and the redundant rosette of the mucosa, which sometimes occurs, when this has not been done, is usually not painful or troublesome and aids materially in the retention of the feces.

The removal of malignant growths of the rectum is now advised in a very large percentage of cases not considered amenable to operation a few years ago.

The technic of these operations is well described in most of our modern textbooks, and need not be repeated. In the review of my own surgical experience I find that I have steadily enlarged the class of cases considered operable and am governed very much more by the evidence that the disease is absolutely local, that is, circumscribed within the periphery of the bowel at its original site of invasion, than by the precise location of the part involved. It is generally recognized that a circumscribed growth, limited to a portion of the bowel within reach of the finger, in either sex, is operable. If there is distinct evidence of glandular infiltration as a secondary development to the primary growth, it is very doubtful if operation is advised. This is the class of cases in which great relief is experienced and life prolonged by a colostomy, since thereby the part affected is given comparative physiologic rest and can be kept from fecal defilement. We are indebted to Kocher for pointing out the very considerable increase of space obtained by the removal of the coccyx.

Little by little portions of the sacrum were removed, and a most noteworthy contribution was given, more especially by Kraske, after whom the 
operation is now named. In a number of instances, I have thought I obtained a decided advantage by first establishing an artificial anus, since by this means the pelvic structures are given a more nearly physiologic rest and can be operated on much more safely. One who has seen, for the first time, the operation for the removal of a considerable segment of the lower bowel performed by a free opening through the sacral region, especially in a moderately thin subject, is surprised at the wide field of operation thus obtained. The bowel may be enucleated with the clear guidance of vision, and even the peritoneal cavity entered from below, freeing the intestines sufficiently to bring down a very considerable loop of the intestine. In this way I have several times resected a portion of the lower bowel, saving the normal anal aperture with the sphincter muscle.

In 1893 I first used a large Murphy button, for the coaptation of the segment, which came away the twelfth day. The thick intestinal wall rendered its application difficult, although the patient did very well. I have since had recourse to suture in addition.

This I believe to have been the first use of the Murphy button in this portion of the bowel, but its applicability was suggested to me by my friend, Dr. H. O. Walker of Detroit. It will frequently be found impossible to save the distal end of the bowel, and then it will be necessary to free the upper portion sufficiently to bring the resected end easily out at the sacral portion of the wound and suture it there. If this has been done aseptically, I think it preferable to close the entire lower segment with lines of buried sutures, thus evenly coaptating the soft parts.

Although the wound is a large one, the vascularization is ample, and the union of the aseptic wound is primal. Special care should be taken in closing the skin with a light-running buried suture which should include only in the deeper portion of it, since otherwise the exceptionally large follicles of the skin may prove a source of infection. This portion of the wound is carefully sealed with iodoform collodion.

Another advantage of this operation, by a wide invasion of the pelvis from below, lies in the possible removal of many of the pelvic glands, a common source of secondary infection. The artificial anus in the sacral region is quite as troublesome as in the iliac region. The mortality following these operations is much less large than even a decade ago, while the results are much more satisfactory. First, because of a better aseptic technic ; and, secondly, because the cases operated upon are brought to the surgeon as soon as the diagnosis has been made, without the fatal delay of tentative treatment which prevailed at an earlier period. The future of this class of operations gives yet better promise, since many details of the surgical technic come within the limit of great possible improvement. It is very probable also that a further specialization of this regional surgery will obtain, since the severer operations are likely to tax the resources of the best surgeons, and a greater familiarity with the local technic aids materially in securing an effective result.

Probably the most valuable contribution to the surgery of the lower bowel is due to the teaching of the gynesic surgeon. The invasion of the abdominal cavity and life-saving results dependent thereon form one of the most brilliant chapters of modern science.

The opening of the abdomen, with the patient in the Trendelenburg position, places the entire pelvic viscera under easy inspection. The small intestines are walled off from above, the peritoneum is opened from below, and the lower portion of the bowel is freed from its attachments. Usually one or two branches of the sacral arteries are divided and require ligature. Then the loop of the intestine is freed, and the diseased portion treated as the conditions demand. If the lower segment is free from disease sufficiently large to permit of its utilization, it should be preserved. If not, it must be removed. Then it will be necessary to doubly ligate the upper portion of the intestine and divide between the ligatures. The upper portion is cleansed and carefully protected from infecting the parts, while the lower segment is entirely removed. This having been done, the bleeding vessels are ligatured, and an artificial anus is made by securing the upper healthy bowel laterally as in a usual colostomy, and the entire pelvic wound is closed with buried sutures, much care being exercised in closing the peritoneum of the pelvic basin. If it has been possible to maintain an aseptic technic, it is better to close the pelvic wound without drainage; otherwise drain. If the upper loop of intestine is of sufficient length, it may be wise to make the artificial anus in the normal site, possibly saving some of the sphincter muscle.

In woman the vagina may be utilized to "piece out" the abbreviated bowel. When this is done much care must be used in closing the pelvic peritoneum about the displaced intestine. When it is possible to resect the diseased portion of the bowel, the intestine is freed as above described, inclosed by ligatures and resected. Reunion of the healthy portions may be effected by retention apparatus, as the Murphy button already referred to, or by sutures. It must be remembered in either case that the connective tissue of the intestine is the important portion for coaptation. Dr. John B. Wyeth of New York, at the present June meeting of the American Medical Association, described a modification of the combined operation, which he had devised and used in three instances.

The sphincter is well dilated and, as far as may be, the lower bowel is emptied and disinfected. The abdomen is entered from above, the peritoneal attachment of the pelvic floor freed, the bowel separated, and the circumscribed portion of the diseased structures tied off and removed as already described. Through the lumen of the lower segment of healthy bowel long forceps are passed, and the upper portion of the freed bowel drawn down externally. This also everts the lower segment and makes easy the careful sutur. 
ing of the two portions of the bowel. After this has been effected, the everted bowel is restored, the bleeding vessels are sutured, the peritoneum of the pelvis is carefully approximated, and the abdominal wound is closed. The perineal wound is drained.

I regard the resection of the lower bowel for cancer by approaching it from above in many instances as a very great advance in modern technic for a variety of reasons. The lymphatic glands of the pelvis can be examined and removed if necessary as by no other route. The resection may be made much more accurately, and in many instances the function of the lower bowel preserved or restored. Cancer of the rectum is one of the most deplorable of all diseases. The last decade has, however, added greatly to the improved surgical measures for its relief and cure, but no field of surgery demands greater improve. ment in skill and technic or promises greater triumph in the relief of suffering.

THE INDICATIONS FOR OPERATION IN MALIGNANT NEOPLASMS OF THE STOMACH. 1

BY CHARLES GREENE CUMSTON, M.D., BOSTON.

Every neoplasm of the stomach should be treated by resection as long as resection is technically possible, and the general condition of the patient is sufficiently satisfactory to allow the operation being done. The presence of evident metastases which cannot be removed does away with all possibility of a durable cure, and constitutes very bad conditions for the immediate success of the operation. These facts are admitted by many surgeons, but are by no means unanimously accepted. Billroth made many reserves in this matter, which Krönlein and Eiselsberg not long ago reviewed in a similar manner. The lattermentioned gentleman says that he never operates on a pyloric carcinoma of any extent in which the fearful symptoms of stenosis are wanting, or where there were only symptoms of cachexia. Billroth believed that a surgical operation would not prolong the life of patients under these circumstances, on account of the natural evolution of carcinoma of the stomach which follows the same course as carcinoma in other organs.

Zawadzki and Solman believe that operation is only indicated in those patients whose general condition depends more on the presence of the mechanical stricture than to the cancerous cachexia. On this last point, however, I would observe that the distinction is not always easy to make, and at the same time the indications for operating, were this rule followed, would be extremely limited indeed.

But the question should be examined in a more general point of view. Now, either the surgeon simply proposes to perform the surgery necessitated by the accidents produced by carcinoma of

"Remarks made in a discussion on "Gastric Cancer" at the annual meeting of the Massachusetts Medical Society, June 11, 1901 . the stomach, in which case the above distinctions would be quite legitimate, or on the other hand, it is the malignant neoplasm itself that he proposes to deal with, in which case the only limits to the feasibility of extirpation of the growth is simply the material impossibility of performing a complete resection far out of the limits of a neoplastic infiltration and in healthy tissues.

Many researches have been undertaken to find out the proportion of operable cases in malignant neoplasms of the stomach, but the larger number of these researches consider only pylorectomy. The first of these were taken from the autopsy records with the following results : In 1876 Gussenbauer and von Winiwarter found, out of 542 carcinomata of the pylorus, that $41.1 \%$ had no metastases, and that $37.7 \%$ had neither metastases nor adhesions. Out of 39 cases, Ledderhose of Strasburg found only $10 \%$ as suitable for operative interference, while Streit of Berne found $25.9 \%$ of his cases could have been successfully operated upon and could have been followed by a cure.

In $1892 \mathrm{~J}$. Lindsay Steven published a paper in the British Medical Journal, in which he records 19 cases of carcinoma of the stomach, 12 of which were localized to the pylorus, 1 at the cardia, and 6 involved the walls of the organ. Of these 19 neoplasms, 6 were free of all adhesions, or $31: 1 \% ; 1$ only presented slight adhesions. Of the 19 , complete absence of metastases existed in 3 cases; in 1 metastasis was only present in the lymphatic glands, while in another the omentum alone was secondarily involved.

The above quotations deserve only a relative merit, because they are simply post-mortem records, and the observations were naturally written by persons who in all probability did not examine the conditions in the surgical point of view. They nevertheless prove that a respectable number of subjects come to the autopsy table in a very satisfactory condition from the surgeon's point of view, and this number, according to the above records, may be placed at about 25 to $35 \%$; or, in other words, from one-fourth to one-third of the total number of patients who die from carcinoma of the stomach.

If we now consult the researches which have been made in the living subject, we find the following: Rydygier, out of 52 patients who had been more or less selected for an operation, only found 5 who did not present metastases or adhesions; in other words, about $9.6 \%$. Krämer, out of 51 cases chosen for operation, found 17 of them without adhesions; in other words, about $33 \%$ suitable for operation. Haberkant found, out of 59 patients who appeared in good condition to undergo an operation, 20 of them were without adhesions, making the operable cases $33.9 \%$. Out of 52 patients, Carle and Fantino found the pylorus free from adhesions in more than $33 \%$ of the cases.

Although these researches are not devoid of interest, still I believe that they only give an inexact idea of what in reality takes place, and I 